[Agr. Biol. Chem., Vol. 36, No. 12, p. 2157 2162, 1972]

\title{
The Formation of Glucose 1,6-Diphosphate from Fructose 1,6-Diphosphate by Yeast Phosphoglucomutase
}

\author{
By Masaaki Hirose, Etsuro Sugimoto and Hideo ChibA \\ Department of Food Science and Technology, Faculty of Agriculture, \\ Kyoto University, Kyoto \\ Received May 12, 1972
}

\begin{abstract}
It was found that fructose 1,6-diphosphate, the main intermediate of glycolysis, was able to act as a coenzyme of yeast phosphoglucomutase reaction. The mechanism of the coenzymatic activity of fructose 1,6-diphosphate was studied. It was indicated in the fructose 1,6diphosphate dependent reaction that glucose 1,6-diphosphate was formed by the phosphatetransfer of fructose 1,6-diphosphate to glucose 1-phosphate in the first step, and in the second step the conversion of glucose 1 -phosphate to glucose 6-phosphate, the original mutase reaction, occurred in the presence of glucose 1,6-diphosphate. The kinetic constants in the reaction of the first step were determined from the time courses of the fructose 1,6-diphosphate dependent reaction.
\end{abstract}

Phosphoglucomutase ( $\alpha$-D-Glucose 1,6-diphosphate: $\alpha$-D-glucose 1-phosphate phosphotransferase, EC 2.7.5.1) catalyzes the reversible interconversion between $\mathrm{G} 1-\mathrm{P}^{*}$ and G6-P, and requires G1,6-diP as a coenzyme. ${ }^{1 \prime}$

$$
\mathrm{G} 1-\mathrm{P}+\mathrm{G} 1,6-\mathrm{diP} \rightleftharpoons \mathrm{G} 1,6-\mathrm{diP}+\mathrm{G} 6-\mathrm{P}
$$

When the substrate is Gl-P, the phosphate group of the 1-position of the coenzyme is transferred to the 6-position of the substrate. The coenzyme is converted to the product, and the substrate, to the coenzyme. ${ }^{2 \prime}$ This interconversion of the substrate and the coenzyme was also represented in phosphoglyceromutase reaction. ${ }^{3)}$ The phosphate groups of the coenzymes must be hydrolyzed in the first step, and in the second step the phosphate must be transferred to the substrate. The phosphatase activities of phosphomutases in the absence of the substrate, which hydrolyze the phosphate group of the

\footnotetext{
* Abbreviations: G1-P, glucose 1-phosphate; G6-P, glucose 6-phosphate; G1,6-diP, glucose 1,6diphosphate; F1,6-diP, fructose 1,6-diphoshate; F1-P, fructose 1-phosphate; F6-P, fructose 6-phosphate.
}

coenzymes, were reported. ${ }^{4 \sim 6)}$ It was confirmed in yeast phosphoglyceromutase that the phosphatase activity was the first step of the mutase reaction. ${ }^{7}$

The mechanisms of the phosphate transfer of the coenzymes to the substrates have been studied. It was reported that the reaction of phosphoglucomutases from rabbit muscle, ${ }^{8 \sim 12)}$ Escherichia coli, ${ }^{13 \sim 14)}$ shark muscle ${ }^{6)}$ and flounder muscle $e^{6}$ proceeded via "ping-pong" pathway, and that the reaction of the enzyme from yeast, ${ }^{151}$ Micrococcus lysodeikticus ${ }^{14}$ and Bacillus cereus, ${ }^{14}$ via "sequential." It was also reported that the reaction of phosphoglyceromutase from rabbit muscle ${ }^{16)}$ proceeded via "ping-pong," and from yeast, ${ }^{17}$, "sequential." These suggested that the pathway of the phosphate transfer of coenzymes in phosphomutases from various origins were not identical. It was shown in the reaction of the rabbit muscle phosphoglucomutase that 1,3diphosphoglycerate ${ }^{18,19)}$ and $F 1,6-$ diP $^{20,21)}$ are also able to act as a coenzyme. This was interpreted by the formation of the enzymephosphate complex, the active intermediate in 
"ping-pong" pathway, from dephosphoenzyme and the diphosphate compounds.

The coenzymatic activity of F1,6-diP was studied in the yeast phosphoglucomutase reaction which proceeded via "sequential" pathway. In this report, the mechanism of coenzymatic activity of F1,6-diP is described.

\section{MATERIALS AND METHODS}

Reagents. G1-P, G6-P, G1,6-diP, F1,6-diP, G6-P dehydrogenase and NADP were purchased from Boehringer Mannheim. G1-P was purified chromatographically by Ray's method ${ }^{22}$ to be freed of G1,6diP. F1,6-diP was used after acid-hydrolysis $(1 \mathrm{~N}$ $\mathrm{HCl}, 100^{\circ} \mathrm{C}, 3 \mathrm{~min}$ ) to exclude the effect of G1,6-diP which might contaminate the F1,6-diP preparation.

Alkali-hydrolyzed coenzymes in Table II were prepared as follows. The coenzyme solutions were heated at $90^{\circ} \mathrm{C}$ in $1 \mathrm{~N} \mathrm{NaOH}$ for $1 \mathrm{hr}$ and were neutralized with Amberlite IR-120 ( $\mathrm{H}^{+}$-form).

Crystalline yeast phosphoglucomutase was prepared as described in the previous paper. ${ }^{153}$

Assay of the enzyme activity. Unless otherwise specified, the reaction mixture contained $0.2 \mu$ mole of G1-P, $5 \mu$ moles of Tris-HCl buffer $(\mathrm{pH} \mathrm{7.5)}$ and various amounts of F1,6-diP or G1,6-diP in a final volume of $0.5 \mathrm{ml}$. The reaction at $25^{\circ} \mathrm{C}$ was started by the addition of the enzyme. After the reaction was stopped by the addition of $3.5 \mathrm{ml}$ of $1.2 \mathrm{~N} \mathrm{H}_{2} \mathrm{SO}_{4}$, the solution was heated at $100^{\circ} \mathrm{C}$ for $3 \mathrm{~min}$. Acid labile phosphate was determined by the method of Chen et al. ${ }^{22}$ )

\section{RESULTS AND DISCUSSION}

Coenzymatic activity of F1,6-diP in the yeast phosphoglucomutase reaction

Coenzymatic activities of acid-hydrolyzed G1,6-diP and F1,6-diP were examined. It was determined from the amounts of inorganic phosphate that G1,6-diP was perfectly hydrolyzed by heating at $100^{\circ} \mathrm{C}$ in $1 \mathrm{~N} \mathrm{HCl}$ for $3 \mathrm{~min}$, and that about 30 percent of F1,6-diP was hydrolyzed under the same conditions. Table I shows that the coenzymatic activity of G1,6-diP was lost by the acid treatment, and
Table I. Coenzymatic Activity of AcidHYDROLYZED F1,6-DIP AND G1,6-DIP

For the measurements of the activity, $0.45 \mu \mathrm{g}$ of the enzyme was used. Acid-hydrolyzed coenzymes were obtained as follows. F1,6-diP was heated at $100^{\circ} \mathrm{C}$ in $1 \mathrm{~N} \mathrm{HCl}$ for $3 \mathrm{~min}$ and neutralyzed with $1 \mathrm{~N} \mathrm{NaOH}$.

\begin{tabular}{llc}
\hline Coenzyme & Treatment & $\begin{array}{c}\text { Activity } \\
(\mathrm{m} \mu \text { mole/min })\end{array}$ \\
\hline $\begin{array}{l}\text { G1,6-diP } \\
1.0 \mu \mathrm{M}\end{array}$ & none & 54.2 \\
\cline { 2 - 3 } & $\begin{array}{l}\text { acid } \\
\text { hydrolysis }\end{array}$ & 0 \\
\hline $\begin{array}{l}\text { F1,6-diP } \\
60 \mu \mathrm{M}\end{array}$ & none & 21.6 \\
\cline { 2 - 3 } & $\begin{array}{l}\text { acid } \\
\text { hydrolysis }\end{array}$ & 19.5 \\
\hline
\end{tabular}

that of F1,6-diP was not. This indicates that the coenzymatic activity of F1,6-diP is not due to G1,6-diP which may be present in the F1,6-diP preparation.

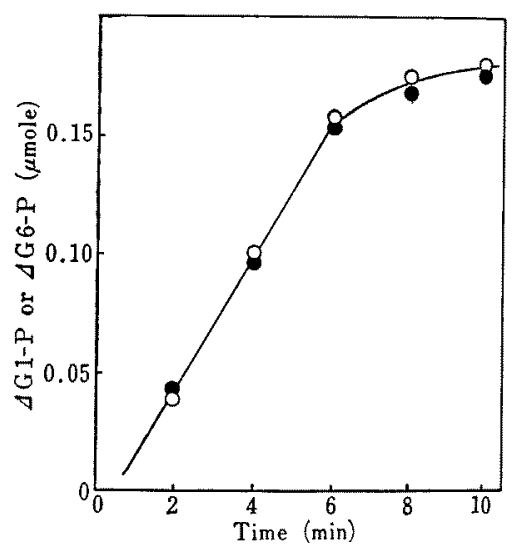

Fig. 1. F1,6-DiP Dependent Reaction of Yeast Phosphoglucomutase.

Reactions were carried out in $0.2 \mu$ mole of G1-P, $0.4 \mu$ mole of F1,6-diP, $20 \mu$ moles of Tris buffer $(\mathrm{pH} 7.5)$ and $0.4 \mu \mathrm{g}$ of the enzyme at $25^{\circ} \mathrm{C}$ in a final volume of $2.0 \mathrm{ml}$. In the determination of G6-P, $20 \mu \mathrm{g}$ of G6-P dehydrogenase and 0.8 $\mu$ mole of NADP were added. The decrease of G1-P was determined by the measurement of acidlabile phosphate, the increase of G6-P, by measuring the absorption of NADPH at $340 \mathrm{~m} \mu$.

Symbols: $\bullet$, Decrease of G1-P; $O$, Increase of G6-P. 
The conversion of G1-P to G6-P was confirmed in the F1,6-diP dependent reaction of the enzyme. G1-P was determined from the amount of acid-labile phosphate, and G6-P, from the amount of NADPH produced by G6-P dehydrogenase. Figure 1 shows that the increase of G6-P agreed well with the decrease of G1-P. This suggests that the main reaction in the $\mathrm{F} 1,6-\mathrm{diP}$ dependent reaction of the enzyme is a conversion of Gl-P to G6-P.

\section{Formation of $G 1,6-d i P$}

The time course of the F1,6-diP dependent reaction was linear after a time lag. This suggested that the two types of the reaction participated in the F1,6-diP dependent reaction. In the second reaction, GI-P was converted to G6-P as shown in Fig. 1. To clarify the first step of the reaction, the enzyme was preincubated with F1,6-diP or with G1-P and F1,6-diP. The reactions were started by the addition of this enzyme solution to the reaction mixture. The time lag was largely

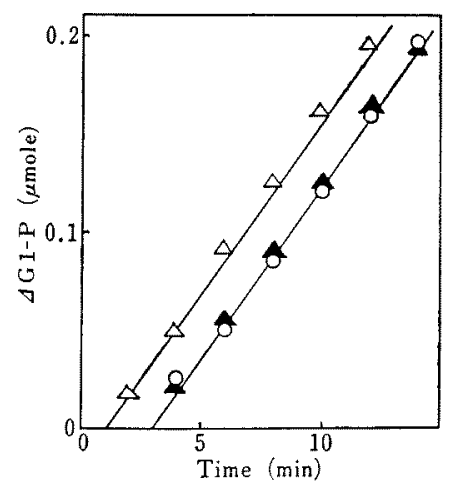

Fig. 2. The Time Lag in the F1,6-DiP Dependent Reaction of the Enzyme.

The reactions were carried out as described in the text. The concentration of F1,6-diP in the reaction mixture was $2.5 \mu \mathrm{M}$. The enzyme was preincubated with F1,6-diP or G1-P and F1,6-diP for $30 \mathrm{~min}$ at $25^{\circ} \mathrm{C}$. Addition to the preincubation mixture:

O, none; $\Delta, 2.5 \mu \mathrm{M}$ F1,6-diP; $\triangle, 2.5 \mu \mathrm{M}$ F1,6-diP and $0.4 \mathrm{mM} \mathrm{G} 1-\mathrm{P}$. diminished only in the case that the enzyme was preincubated with G1-P and F1,6-diP as shown in Fig. 2. This suggests that in the first step of the reaction the enzyme-phosphate complex is not formed, but the direct phosphate transfer of F1,6-diP to G1-P occurs as shown in the reaction (b).

$$
\begin{aligned}
& \mathrm{G} 1-\mathrm{P}+\mathrm{F} 1,6-\mathrm{diP} \rightleftharpoons \\
& \quad \mathrm{G} 1,6-\mathrm{diP}+\mathrm{F} 6-\mathrm{P} \text { (or F1-P) }
\end{aligned}
$$

This reaction is reasonably interpreted by "sequential" pathway in the yeast phosphoglucomutase reaction.

The formation of G1,6-diP in the reaction (b) was confirmed as follows. F1,6-diP is destroyed by alkali-treatment, and G1,6-diP is relatively stable under the treatment. F1,6diP was incubated with the enzyme or with G1-P and the enzyme. After an alkalitreatment of the solutions, the coenzymatic activities were measured. Table II shows that the coenzymatic activity is not lost in the case that F1,6-diP is incubated with G1-P and the enzyme. These indicate that yeast phosphoglucomutase is able to catalyze the reaction (b).

\section{Kinetic constants}

Figure 3 shows that the velocities of F1,6diP dependent reaction is increased, and the time lag is decreased with an increment of the concentration of F1,6-diP added. LineweaverBurk plot of $1 / r_{\mathrm{app}}$ versus $1 /[\mathrm{F} 1,6-\mathrm{diP}]$ was linear as shown in Fig. 4. The apparent velocity was obtained from the slope of linear increase in Fig. 3.

The $v_{a p p}$ values should be the velocities of the reaction (a) in the presence of G1,6-diP which is formed in the reaction (b) as concluded from Fig. 1 and Table II. Thus the concentration of G1,6-diP $x$, formed within the time lag can be calculated from the following equation (1).

$$
v_{\mathrm{app}}=\frac{x \cdot V_{\mathrm{M}, \mathrm{g}}}{K m_{\mathrm{g}}+x}
$$


Table II. Coenzymatic Activity of Alkali-hydrolyzed F1,6-DIP AND G1,6-DIP

The coenzymatic activity of F1,6-diP and G1,6-diP were measured with use of $0.54 \mu \mathrm{g}$ of the enzyme after the alkali-hydrolysis of the coenzymes which were previously incubated at $25^{\circ} \mathrm{C}$ with $0.50 \mu \mathrm{g}$ of the enzyme and $1.2 \mu$ moles G1-P in a volume of $3.0 \mathrm{ml}$. Alkali-hydrolysis was carried out as described in the text.

\begin{tabular}{ccccc}
\hline \multirow{2}{*}{ Coenzyme } & \multicolumn{2}{c}{ Preincubation } & Treatment & $\begin{array}{c}\text { Activity } \\
\text { (mumole/min) }\end{array}$ \\
\cline { 2 - 3 } & Addition & Time (min) & & None \\
F1,6-diP & None & - & & 19.0 \\
$(10 \mu \mathrm{M})$ & None & - & Alkali & 0 \\
& Enzyme & 75 & Alkali & 0 \\
& Enzyme, G1-P & 0 & Alkali & 0 \\
& Enzyme, G1-P & 75 & Alkali & 12.1 \\
\hline $\mathrm{G} 1,6-\mathrm{diP}$ & None & - & None & 43.0 \\
$(0.2 \mu \mathrm{M})$ & Enzyme, G1-P & 75 & Alkali & 22.0 \\
\hline
\end{tabular}

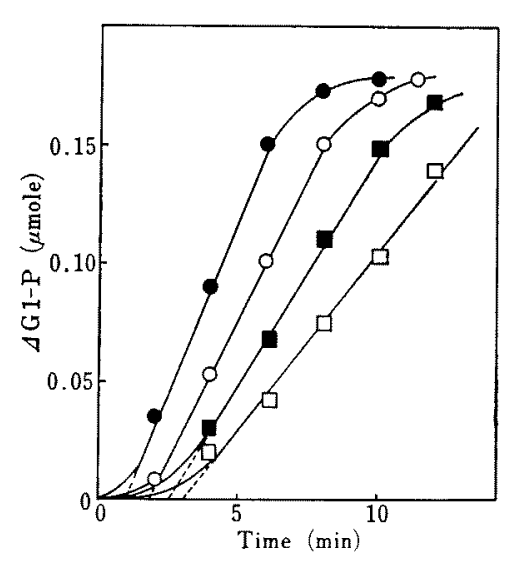

FIG. 3. Time Courses of F1,6-DiP Dependent Reaction.

The reactions were carried out as described in the text. Concentrations of F1,6-diP:

$\bullet 0.10 \mathrm{mM} ; 0,10 \mu \mathrm{M} ; \mathbf{a}, 5.0 \mu \mathrm{M} ; \square, 2.5 \mu \mathrm{M}$.

Where $V_{M, g}$ and $K m_{s}$ are the maximum velocity and $K m$ value for G1,6-diP $(0.14$ $\left.\mu \mathrm{M}^{15}\right)$ in the $\mathrm{G} 1,6$-diP dependent reaction, respectively. Therefore, the velocity of the reaction (b), $v_{f}$, can be obtained from the equation (2).

$$
v_{i}=\frac{x}{T}
$$

Where $T$ represents the time lag in the F1,6-

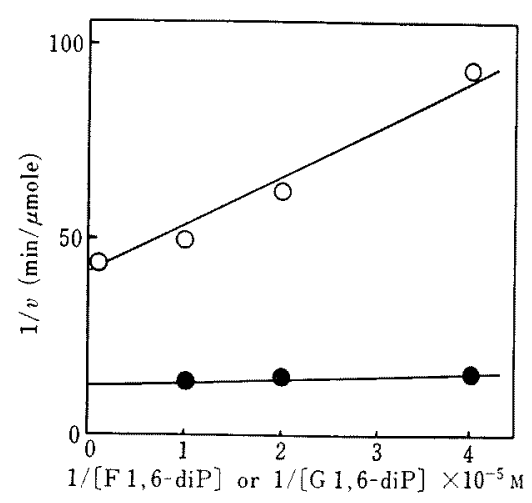

Fig. 4. Lineweaver-Burk Plot of Yeast Phosphoglucomutase Reaction.

The reactions were carried out as described in the text. Symbols:

O, F1,6-diP; •, G1,6-diP.

diP dependent reaction. The values of $T$ were obtained from the intercepts of the dotted lines on the abscissa in Fig. 3, and $x$, from Fig. 4 with use of the equation (1). The values of $v_{f}$ were calculated from the equation (2). Figure 5 shows that the LineweaverBurk plot of $1 / v_{\mathrm{f}}$ versus $1 /[\mathrm{F} 1,6$-diP] is linear. The $K m_{\mathrm{f}}$ value, $K m$ for F1,6-diP in the reaction (b), was $40 \mu \mathrm{M}$, and $V_{\mathrm{M}, \mathrm{f}}$, the maximum velocity in the reaction (b), was $0.04 \mathrm{~m} \mu \mathrm{mole} /$ 


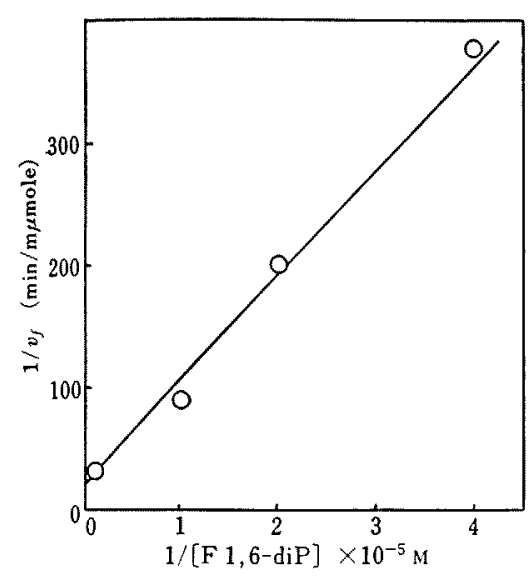

Fig. 5. Lineweaver-Burk Plot of $1 / \mathrm{v}_{\mathrm{f}}$ versus $1 /$ [F1,6-diP].

The values of $v_{f}$ were calculated from the equation (2) in the text.

$\min$. These indicate that $K m_{\mathrm{f}} / K m_{\mathrm{g}}$ is 290 and $V_{\mathbb{X}, \mathrm{I}} / V_{\mathrm{M}, \mathrm{g}}$ is $1 / 530$ (see Fig. 4).

It was demonstrated that in the F1,6-diP dependent reaction of the enzyme, the slow reaction (b) occurred in the first step, and the fast reaction (a), in the second step. The time course of the overall reaction is theoretically shown as follows. The velocity of formation of G1,6-diP, $d x / d t$, in the reaction (b) is shown as the equation (3), since F1,6diP should bind to the G1,6-diP binding site of the enzyme.

$$
\frac{d x}{d t}=\frac{\left([F]_{0}-x\right) V_{\mathrm{M}, \mathrm{f}}}{\left([F]_{0}-x\right)+K m_{\mathrm{f}}\left(1+x / K m_{\mathrm{g}}\right)}
$$

Where $[F]_{0}$ represents the initial concentration of F1,6-diP. The equation (4) is obtained by intergration of the equation (3).

$$
\begin{aligned}
& \left(1-\frac{K m_{\mathrm{f}}}{K m_{\mathrm{g}}}\right) x-K m_{\mathrm{f}}\left(1+\frac{[F]_{0}}{K m_{\mathrm{g}}}\right) \\
& \times \ln \left(1-\frac{x}{[F]_{0}}\right)=V_{\mathrm{M}, \mathrm{f}} \cdot t
\end{aligned}
$$

The equation (4) is simplified as follows, since $[F]_{0}$ and $K m_{\mathrm{f}}$ are large enough compared with $K m_{\mathrm{g}}$.

$$
-\frac{x}{[F]_{0}}-\ln \left(1-\frac{x}{[F]_{0}}\right)=\frac{V_{\mathrm{M}, \mathrm{f}} \cdot K m_{\mathrm{g}}}{[F]_{0} \cdot K m_{\mathrm{f}}} \cdot t
$$

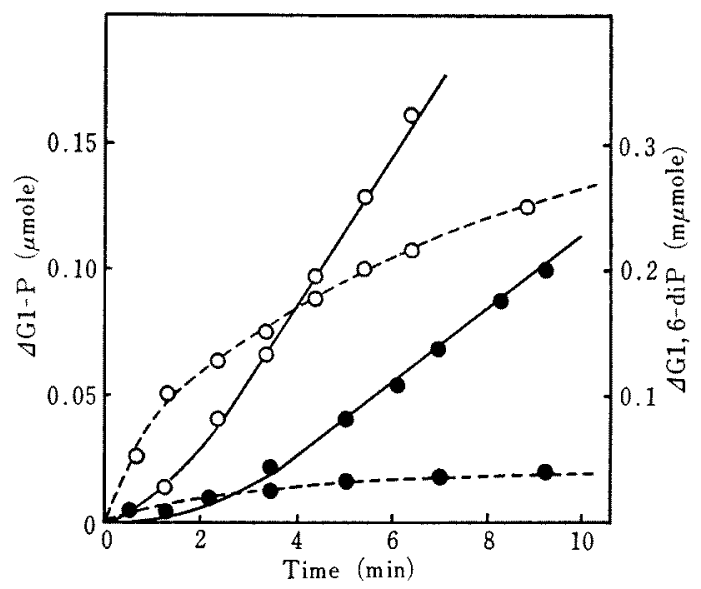

FIG. 6. Theoretical Time Courses of F1,6-DiP Dependent Reaction.

The solid lines and the dotted lines represent the decrease of G1-P and the increase of G1,6-diP, respectively. The concentration of F1,6-diP:

$$
0,0.1 \mathrm{~mm} ; \bullet, 2.5 \mu \mathrm{M} \text {. }
$$

Figure 6 shows that the theoretical time courses calculated from the constants obtained from the experimental data are linear after a time lag, in spite of the fact that the amount of G1,6-diP increases with a progress of time. The time courses in Fig. 6 agreed well with Fig. 3. This indicates that the F1,6-diP dependent reaction of the enzyme is due to the reaction (a) and (b).

The reaction (b) is important for a sugar metabolism of yeast on the point that G1,6diP of the coenzyme of phosphoglucomutase can be produced from F1,6-diP, the main intermediate of glycolysis, by the enzyme. It is considered that the reaction (b) acts as the pathway of the G1,6-diP biosynthesis as well as the phosphoglucokinase reaction. ${ }^{23}$,

\section{REFERENCES}

1) L. F. Leloir, R. E. Trucco, C. F. Cardini, A. C. 
Paladini and R. Coputto, Arch. Biochem., 19, 339 (1948).

2) E. W. Sutherland, M. Cohn, T. Posternak and C. F. Cori, J. Biol. Chem., 180, 1285 (1949).

3) E. W. Sutherland, T. Posternak and C. F. Cori, ibid., 181, 153 (1949).

4) B. K. Joyce and S. Grisolia, ibid., 233, 350 (1958).

5) R. Sasaki, E. Sugimoto and H. Chiba, Arch. Biochem. Biophys., 115, 58 (1966).

6) T. Hashimoto and P. Handler, J. Biol. Chem., 241, 3940 (1966).

7) R. Sasaki, M. Hirose, E. Sugimoto and H. Chiba, Biochim. Biophys. Acta, 227, 595 (1971).

8) V.A. Najjar and M. E. Pullman, Science, 119, 631 (1954).

9) J. B. Sidbury, Jr. and V. A. Najjar, J. Biol. Chem., 227, 517 (1957).

10) E. P. Kennedy and D. E. Koshland, Jr., ibid., 228, 419 (1957).

11) J. A. Yankeelov, Jr., H. R. Horton and D. E. Koshland, Jr., Biochemistry, 3, 349 (1964).

12) W. J. Ray, Jr., and G. A. Roscelli, J. Biol. Chem., 239, 1228 (1964).
13) J. B. Joshi and P. Handler, J. Biol. Chem., 239, 2741 (1964).

14) K. Hanabusa, H. W. Dougherty, C. Del Rio, T. Hashimoto and P. Handler, ibid., 241, 3930 (1966).

15) M. Hirose, E. Sugimoto, R. Sasaki and H. Chiba, J. Biochem., 68, 449 (1970).

16) Z. B. Rose, Arch. Biochem. Biophys., 140, 508 (1970).

17) H. Chiba, E. Sugimoto, R. Sasaki and M. Hirose, Agr. Biol. Chem., 34, 498 (1970).

18) J. B. Alpers, J. Biol. Chem., 243, 1698 (1968).

19) J. B. Alpers and G. K. H. Lam, ibid., 244, 200 (1969).

20) J. V. Passonneau, O. H. Lowry, D. W. Schults and J. G. Brown, ibid., 244, 902 (1969).

21) O. H. Lowry and J. V. Passonneau, ibid., 244, 910 (1969).

22) P. S. Chen, Jr., T. Y. Toribara and H. Warner, Anal. Chem., 28, 1756 (1956).

23) A. C. Paladini, R. Copputto, L. F. Leloir, R. E. Trucco and C. E. Cardini, Arch. Biochem., 23, 55 (1949). 